Cinémas

Revue d'études cinématographiques

Journal of Film Studies

\title{
Le spectacle du " manque féminin » au cinéma : un leurre qui en cache un autre
}

\section{Denise Pérusse}

Volume 8, numéro 1-2, automne 1997

Cinéma et mélancolie

URI : https://id.erudit.org/iderudit/024743ar

DOI : https://doi.org/10.7202/024743ar

Aller au sommaire du numéro

Éditeur(s)

Cinémas

ISSN

1181-6945 (imprimé)

1705-6500 (numérique)

Découvrir la revue

Citer cet article

Pérusse, D. (1997). Le spectacle du « manque féminin » au cinéma : un leurre qui en cache un autre. Cinémas, 8(1-2), 67-91. https://doi.org/10.7202/024743ar

\section{Résumé de l'article}

À partir d'une anthologie de textes féministes fondateurs en études cinématographiques, l'auteure fait ressortir tout un paradigme de négativités autour de l'image de la femme dans le cinéma classique et de l'identité de la spectatrice. Elle montre aussi comment une théoricienne comme Kaja Silverman ouvre une brèche dans les théories du genre en montrant comment les femmes dans le cinéma classique sont contraintes d'exhiber le manque afin d'absorber les pertes qui affectent le sujet masculin. 


\title{
Le spectacle $\mathrm{du}$ "manque féminin " au cinéma: un leurre qui en cache un autre
}

\section{Denise Pérusse}

\begin{abstract}
RÉSUMÉ
A partir d'une anthologie de textes féministes fondateurs en śtudes cinématographiques, l'auteure fait ressortir tout un paradigme de négativités autour de l'image cle la femme dans le cinéma classique et de Pidentité de la spectatrice. Elle montre aussi comment une theoricienne comme Kaja Silverman ouvre une brèche dans les théories du genre en montrant comment les femmes dans le cinéma classique sont contraintes d'exhiber le manque afin d'absorber les pertes qui affectent le sujet masculin.
\end{abstract}

\section{ABSTRACT}

On the basis of an anthology of founding texts in feminist film studies, the author puts forward a paradigm of negativities surrounding the image of women in classical cinema and the identity of the female spectator. She also shows how a theoretician like Kaja Silverman opened a breech in theories of genre by showing how women in classic cinema are forced to exhibit their own lack in order to absorb the losses which affect the masculine subject.

La théorie filmique a été hantée depuis son tout début par le spectre de l'absence inscrit au cour même du dispositif cinématographique. Le cinéma est l'histoire de rencontres manquées, 
"[...] d'un rendez-vous manqué entre le voyeur et l'exhibitionniste», affirmait Christian Metz (1993). Le film communique ses illusions à travers d'autres illusions, bref repose sur l'absence de l'objet représenté. Le spectateur / la spectatrice reçoit des images qui se donnent comme la représentation de bien plus que ce qu'elles sont; il confond représentation et perception et prend la fiction pour la réalité. Pourtant, le spectateur n'est pas dupe de l'illusion diégétique, sait fort bien que l'écran ne représente rien d'autre qu'une fiction et malgré tout, précise Denis Bellemare, «[...] il y adhère quand même comme réalité qui se donne à voir comme un réseau hermétiquement mobile de caches, d'entre-deux, d'entre-plans, un jeu d'absence et de présence» (1992, p. 143).

Metz envisage cette perte de l'objet, du réel comme une castration. Ainsi, le sujet de la réception (homme ou femme) se protège-t-il du traumatisme de la castration en ayant recours à des mécanismes de défense similaires à ceux adoptés par l'enfant, soit le désaveu et le fétichisme. Comme le petit garçon chez Freud qui voit pour la première fois les organes génitaux féminins et qui désavoue l'absence de pénis, le sujet spectatoriel refuse de reconnaître ce qu'il perçoit pourtant très bien. $\mathrm{Ce}$ désaveu est conforté par la construction d'un fétiche. Lorsqu'il voit un film, le spectateur se met à l'abri de cette perception du manque en prenant un substitut à la place du réel absent. Ce substitut s'impose comme une condition préalable au plaisir. $\mathrm{Ce}$ substitut, nous le verrons plus loin, s'avérera l'enjeu même de la représentation au négatif de la femme au cinéma.

Il y a aussi une autre condition au plaisir de regarder: garder ses distances. Le fétichiste comme le voyeur... comme le spectateur doit maintenir une distance avec l'image. Le plaisir visuel au cinéma implique donc un processus d'objectivation de l'image, de séparation entre le sujet et l'objet du regard. Par ailleurs, si le sujet spectatoriel fait semblant, sachant fort bien qu'il est au cinéma, bref s'il garde ses distances, les images et les sons qui défilent lui offrent un espace animé et habité par des corps similaires au sien, corps auxquels il peut néanmoins, paradoxalement, s'identifier. Cela tend à réduire l'écart entre lui et l'écran et, en conséquence, à masquer l'absence sur laquelle est fondée 
la représentation cinématographique. Cet investissement spectatoriel, cette identification impliquent une fusion du sujet à l'objet du regard, fusion qui se développe à travers le narcissisme et la constitution du Moi (ce que Lacan a appelé l'expérience du miroir '). Bref, le corps des personnages joue comme leurre perceptuel et comme point d'ancrage de l'identification.

Si la psychanalyse appliquée à la théorie filmique a su rendre compte de l'absence de l'objet dans l'expérience cinématographique aussi bien que des défenses du spectateur face à cette absence, peu de théoriciens ont tissé les liens entre la perte de l'objet et le travail de l'absence qui structurent le cinéma, la représentation de la femme comme manque et sa position comme spectatrice. Cette omission, les théoriciennes féministes se sont chargées de la combler. Dans le cadre de ce présent article, je tenterai d'embrasser en quelques pages (véritable tour de force s'il en est!) toute une série de textes féministes fondateurs en études cinématographiques qui se sont arrimés à la psychanalyse et ont participé à une meilleure connaissance du signifiant cinématographique, du travail spécifique du cinéma classique et des différentes strates de négativité mises en place autour du personnage principal, "Elle», cette porteuse d'un "imaginaire en négatif " au même titre que le film, pour paraphraser Denis Bellemare $^{2}$. Cette analyse des textes non seulement tient compte de leur chronologie, mais encore cette chronologie est à même de démontrer et de démonter un parcours significatif qui passe d'une position unique à multiple, fixe à mobile.

Tout en démélant cet écheveau de négativités, de négations, d'exclusions autour de l'image de la femme dans le cinéma classique $^{3}, \mathrm{j}^{\mathrm{j}}$ aborderai au passage comment les théories du genre, en s'attardant notamment à un corpus hollywoodien centré sur des personnages féminins forts, éclairent certains aspects du travail de la mélancolie intimement liés à la posture identificatoire du sujet de la réception comme la perte de l'objet, l'ambivalence et la régression narcissique.

\section{La femme comme signe de turbulence}

Pour éprouver leurs hypothèses, les théoriciennes féministes ont appelé à la barre des films du corpus narratif classique, 
objets de fascination mais aussi de frustration. Objets de fascination en ce qu'ils demeurent un terrain extrêmement riche et changeant; objets de frustration parce qu'elles n'y trouvent jamais réfléchies par l'écran des femmes comme sujets à part entière. Dans le mode traditionnel de représentation de la féminité, il semble qu'il faille comprendre l'image de la femme comme un signifiant dont la fonction est de représenter le désir masculin. Les personnages féminins ne peuvent qu'être regardés ou subir. Pire, quand c'est une femme qui s'approprie le regard, "supporte le désir", elle devient presque inévitablement une figure tragique punie par le récit. La femme en tant que sujet désirant est pratiquement un signe impossible.

Dès le départ, la théorie féministe filmique a inscrit la différence sexuelle au cœur du dispositif cinématographique et s'est attardée à l'image en creux de la femme dans le cinéma classique. Parmi celles qui ont secoué les études féministes, il y a bien évidemment la pionnière britannique Laura Mulvey. Paru en 1975, l'article de Mulvey, "Visual Pleasure and Narrative Cinema ", a joué un rôle catalyseur dans la réflexion théorique sur la différence sexuelle au cinéma et la relation du sujet spectatoriel au film.

S'appuyant sur des prémisses freudiennes et lacaniennes, Mulvey montre que le cinéma dominant repose sur l'image de la femme castrée. Posant une nette dichotomie sexuelle au cœur du dispositif visuel (les hommes regardent; les femmes sont l'objet du regard), elle dévoile le potentiel traumatique que suppose le spectacle de la femme comme manque et les moyens de défense donnés au spectateur pour y faire face. Le scénario décrit par Mulvey est beaucoup plus conforme au paradigme psychanalytique originel de castration et de crise que celui de Metz. Ce dernier conceptualise la castration à partir du modèle lacanien où la castration est vue comme un ensemble de divisions à travers lesquelles le sujet est constitué, divisions qui précèdent le récit œdipien et la différence sexuelle. La castration désigne donc la perte de "l'objet" provoquée par le signifiant cinématographique, une perte qui donne une signification aux divisions que connaît le sujet (divisions amorcées par le stade du miroir et l'entrée dans le langage). Cette façon de conceptualiser 
la castration a été grandement critiquée par Jacqueline Rose (1986), entre autres, qui y voit une façon d'éviter d'aborder la différence sexuelle au cinéma. Aussi insiste-t-elle pour qu'on revienne au concept freudien de désaveu pour comprendre comment l'image de la femme au cinéma se construit autour de la référence phallique.

Donc, la tension reconnue par Freud entre le plaisir de regarder, ce qu'il a appelé la scopophilie ou le voyeurisme, et le danger représenté par l'évocation du complexe de castration se jouera à travers l'image de la femme exhibée pour le plaisir du spectateur masculin. Le modèle se présente comme suit : comme le petit garçon, le spectateur qui regarde une femme au cinéma se sent menacé et craint d'être, comme elle, privé de pénis. Aussi, le cinéma classique offre deux possibilités pour résoudre cette crainte de la castration:

- le désaveu à travers le fétichisme (la femme est fétichisée afin de la rendre rassurante plutôt que dangereuse; un vêtement, une partie du corps féminin devient le lieu d'un investissement compensatoire et remplace l'organe manquant);

- la "prise de distance" (le héros masculin qui fonctionne comme le Moi idéal du spectateur subjugue la femme, la possède ou la punit de façon sadique, diminuant ainsi la menace sexuelle qu'elle représente; le spectateur masculin prend ses distances avec le "spectacle du manque" et adopte une attitude méprisante envers la «créature mutilée" qui est son "pendant " sexuel).

Le mode de représentation de la femme satisfait le plaisir voyeuriste du spectateur masculin et les conventions du récit permettent aussi de satisfaire son narcissisme. En s'identifiant à son semblable au sein de la diégèse, celui-ci peut posséder le personnage féminin dépeint comme objet sexuel. Autrement dit, le film narratif classique est conçu pour stimuler le plaisir de regarder du sujet masculin (à la fois dans le film et dans la salle de cinéma), la femme étant connotée comme objet du regard, sans plus.

S'appuyant sur les films d'Alfred Hitchcock et de Josef von Sternberg, qui font appel à toute une chaîne de négations et 
d'exclusions, Laura Mulvey conclut du voyeurisme cinématographique et de la nature du regard que le film s'adresse à un sujet spectatoriel exclusivement de sexe masculin, la spectatrice étant conçue comme absence, comme lieu d'une impossibilité. Pour la théoricienne, les femmes n'auraient pas grand-chose à regretter de la mort du cinéma classique. Je dirais même plus: il faudrait, selon elle, en arriver à détruire le plaisir narratif établi par la tradition représentative réaliste - plaisir basé sur l'activité voyeuriste lequel s'est manifesté historiquement dans l'image de la femme - et à concevoir un nouveau langage pour le désir.

Les travaux des théoriciennes britanniques comme Claire Johnston et Pam Cook parus à la même époque analysent également la présence de la femme à l'écran comme signe de turbulence, spectacle provoquant une véritable anxiété chez l'homme et devant, de ce fait, être contenu. Comme pour Mulvey, le cinéma narratif classique, entendre ici à titre exemplaire le cinéma de Raoul Walsh, place le spectateur dans une position spécifique et encourage une lecture fétichiste. Tout un paradigme de négativités instaure la présence de la femme à l'écran, qui apparaît alors comme l'effet d'une absence. La figure féminine à l'écran n'a d'existence qu'à travers les discours ou les actions des personnages masculins. Sa présence ne fait que célébrer sa non-existence. Elle demeure une figure en creux, un signe qui est défini négativement, par son manque (la femme comme être castré, comme non masculine), manque qui doit être repéré, contenu, situé de sorte que les visées narcissiques de l'homme puissent se réaliser ${ }^{4}$.

Pourtant, même dans les films de Walsh, on trouve des moments où les personnages féminins résistent à cette réduction au statut de signe d'échange pour l'homme. Ces moments créent un désordre textuel et rendent le discours masculin « fragmenté", incohérent. Ces brèches sont encore plus apparentes dans les films de Dorothy Arzner, une des rares réalisatrices à avoir percé Hollywood dans les années trente et quarante. Dance, Girl, Dance (1940) est immanquablement appelé à la barre. Dans ce film qui raconte la vie et les déboires d'une troupe de danseuses, l'une des danseuses fait face à un auditoire masculin enragé et explique aux hommes "comment elle les 


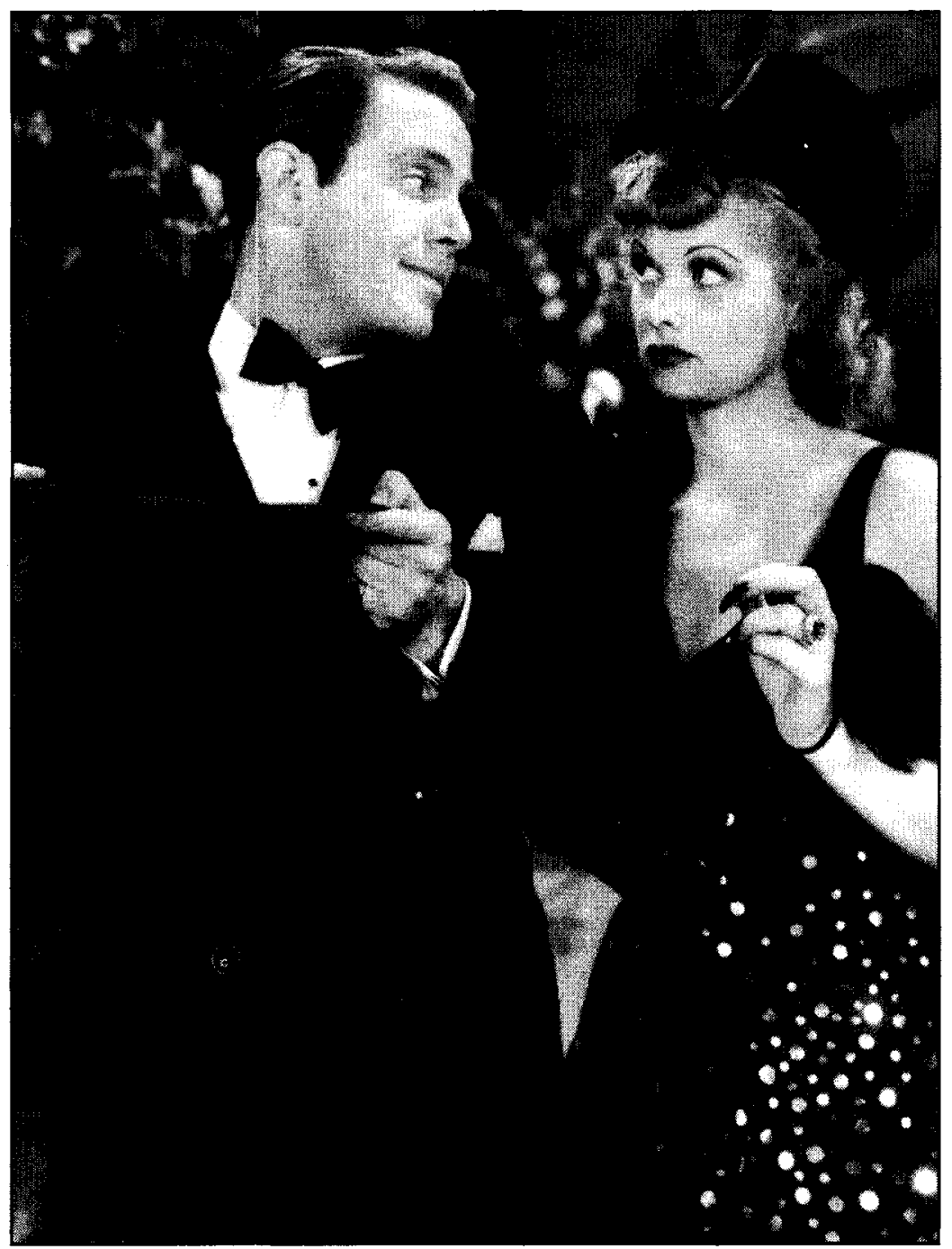

\section{Dance, Girl, Dance de Dorothy Arzner (1940)}

Collection Cinémathèque québécoise

voit ${ }^{5} "$. Ce renvoi du regard dans un film où il est censé être à sens unique constitue une attaque directe à la fois du public diégétique et de celui du film, attaque qui déstabilise la représentation des femmes comme spectacle, comme objet de désir. Ce renvoi spéculaire prend la forme d'une instabilité symptomatique qui autorise une lecture contre le grain du texte, en 
questionne l'unité, la cohérence, et permet un déplacement de l'identification. Un film comme Dance, Girl, Dance déstabilise partiellement le sujet spectatoriel et crée une tension entre le désir du personnage féminin et un système de représentation qui la positionne comme objet de désir. Dans les films d'Arzner donc, c'est l'univers masculin qui est amené à être examiné, "qui est rendu étrange».

Cette lecture à contre-courant inspirera la théorie féministe des années quatre-vingt qui introduira les concepts de progressive ou subversive reading, de resisting reader (c'est-à-dire un sujet spectatoriel qui refuse de prendre la place que lui offre le texte filmique par opposition au spectateur idéal construit par ce même texte). Jackie Byars (1988), par exemple, montrera qu'un nombre significatif de récits hollywoodiens, notamment les mélodrames familiaux centrés sur des femmes, laissent place à de véritables luttes idéologiques, ouvrent une brèche pour une voix féminine et offrent une variété de positions identificatoires. Pour elle, il n'y a pas de textes idéologiquement cohérents, ni monolithiquement répressifs, ni de sujet spectatoriel invariablement masculin.

\section{La femme comme pivot dans la mise en scène du désir}

Si Mulvey comme Cook et Johnston conçoivent la figure féminine à l'écran comme un signe de turbulence, comme le symptôme d'un malaise qu'il faut repérer et contenir, les théoriciens comme Raymond Bellour, Thierry Kuntzel et Stephen Heath ont remis en question cette position. Dans leurs tentatives de décrire systématiquement le travail du film narratif, ils ont mis en évidence la tendance du récit classique à tabler sur la répétition, la redondance, la symétrie, mais aussi à "se rompre" pour ensuite aplanir, résoudre les divisions, les différences, les contradictions. Donc, les écarts, les «lézardes» dans un film classique que les féministes ont attribués aux difficultés imputées à l'image de la femme comme manque ou aux tentatives de mettre en scène un désir féminin dans un modèle patriarcal n'étaient rien de plus pour Bellour et consorts que les composantes essentielles de la structure du film classique.

Les analyses textuelles détaillées que Bellour fait des structures de fascination dans le film classique, notamment Les Oiseaux ${ }^{6}$, 
ont retenu l'attention des théoriciennes féministes de Camera Obscura ${ }^{7}$, car elles éclairent la façon dont la signification est produite dans le film classique de même que la fonction et la position de la femme dans la mise en scène du désir, mise en scène implacable qui repose essentiellement sur le regard, pièce mâ̂tresse au cinéma ${ }^{8}$. Son analyse s'avère plus complexe que celle de Mulvey en ce qu'elle focalise sur le désir inscrit dans le regard des femmes (Melanie Daniels dans le cas des Oiseaux) - qui sera l'objet d'un examen d'Hitchcock et de ses délégués fictionnels (Mitch Brenner) — plutôt que sur le corps des femmes comme objet scopique ou fétiche. Toujours pour Bellour, un regard, un désir féminin est nécessaire à la fiction, mais seulement parce qu'il sera finalement puni, circonscrit, contrôlé ou assimilé au désir du héros (masculin) et, par extension, du spectateur (masculin).

Dans son analyse, le théoricien tente également de trouver une place à la spectatrice contrairement à Mulvey pour qui le spectateur ne peut être que masculin. Il reconnaît qu'il est compréhensible que les femmes ne trouvent pas leur compte dans un système de représentation insistant sur leur absence bien qu'elles soient présentes. Mais il importe selon lui que les femmes se demandent ce qui semble les attirer si fortement dans ces films. Bellour insiste sur la nature masochiste du plaisir que peut retirer la spectatrice en les visionnant.

A la suite de cette description détaillée du film classique qui n'accorde aucune place aux désirs féminins, ni de position à la spectatrice, sinon une position masochiste comme chez Bellour, bref, face à ce modèle du cinéma classique aussi totalisant et déterministe que celui de Mulvey, que peuvent donc répliquer les critiques féministes? Comme le précise Jackie Stacey, le hic chez Bellour, c'est qu'il caractérise la position des spectateurs en la calquant sur une dichotomie manichéenne associant la différence sexuelle masculin/féminin au couple antinomique sadisme/masochisme qu'il croit mis en scène par le dispositif cinématographique. Le spectateur y est contraint par le texte filmique à une identification sexuelle prédéterminée. Il n'y a pas de place pour un processus d'identification ou pour un choix d'objet changeant, précaire. Autrement dit, les femmes prennent-elles nécessairement 
une position spectatorielle féminine et les hommes une position masculine ${ }^{\text {? }}$ ?

\section{Le travestissement comme mode d'accès au désir}

En 1981, Laura Mulvey revient sur cette question qu'elle avait occultée dans "Visual Pleasure and Narrative Cinema", à savoir la place de la spectatrice et plus spécifiquement les types d'identification dans les films où le personnage féminin est au cour du récit. Dans une tentative de développer une position plus mobile pour la spectatrice, Mulvey se tourne vers les théories de la féminité de Freud selon lesquelles la jeune fille doit passer à travers une phase masculine active avant d'adopter une position féminine correcte. Contraintes d'abandonner leur masculinité, leurs fantasmes d'action et l'objet féminin de leur prime enfance, les femmes sont déchirées entre une féminité passive et une masculinité régressive. Mulvey prend comme exemple Duel in the Sun, un film où l'héroïne est incapable de réaliser une identité sexuelle stable ${ }^{10}$.

Le dilemme vécu par le personnage féminin est partagé par la spectatrice. Dans ce film, il est vraisemblable que la spectatrice s'identifie tantôt au personnage féminin, adoptant une position passive dite masochiste, tantôt au personnage masculin, réactivant ses fantasmes d'action que réprime la féminité sanctionnée par le patriarcat. Mulvey montre comment un tel film dirige la réception vers autre chose que la castration, l'absence ou la trajectoire odipienne et qu'il y est surtout question de désirs conflictuels chez la femme. La femme n'est plus conçue comme absence; elle est présente mais problématique, la féminité étant vue comme un site instable, contradictoire.

L'intérêt de cette nouvelle analyse de Mulvey réside dans le fait qu'elle ne pose plus une position spectatorielle fixe construite par le texte filmique, à savoir un modèle spectatoriel unifié et masculin. Cependant, elle maintient que les fantasmes d'action ne peuvent trouver expression qu'à travers une figure de travesti. Afin de s'identifier au désir actif, la spectatrice doit trahir son sexe et se positionner comme si elle était un homme, bref elle est condamnée à un certain travestissement, position par ailleurs inconfortable, où elle se sent mal à l'aise. Le travestissement, le 


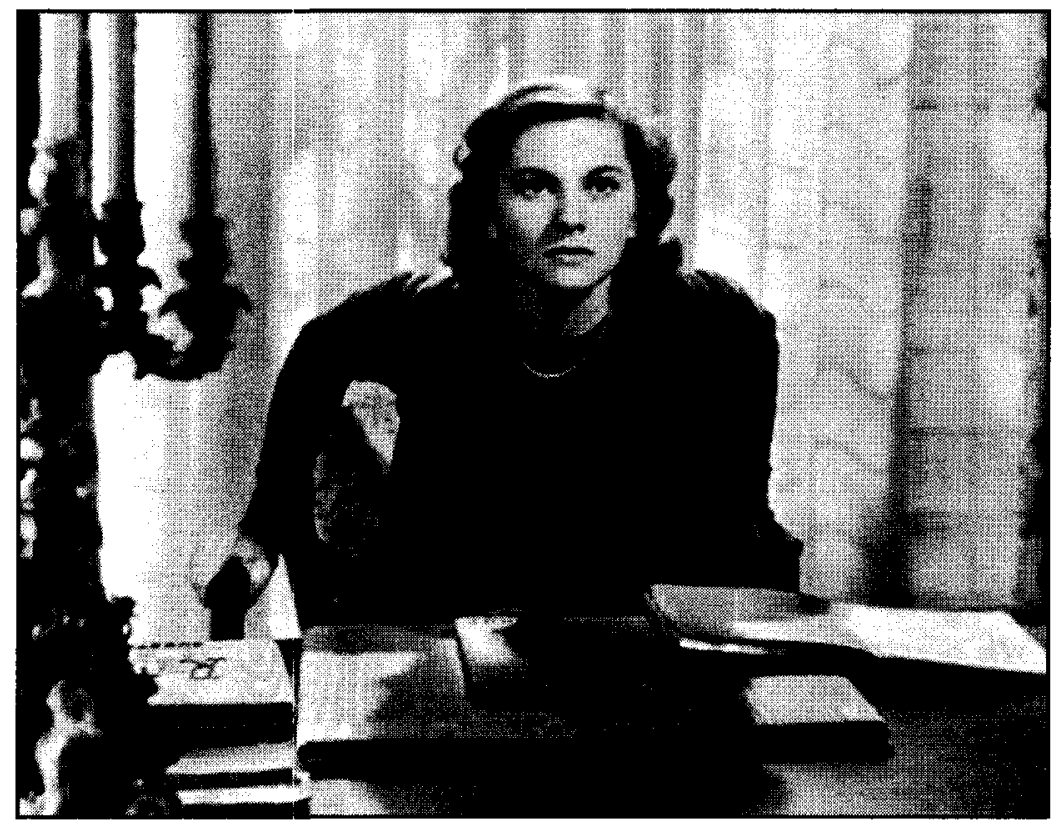

\section{Rebecca \\ d'Alfred Hitchcock (1940)}

Collection Cinémathèque québécoise

fait de porter les attributs des personnages d'un autre sexe, est une occasion de clésir pour la femme, car cela lui permet de mâ̂triser l'image, de s'en distancier, une condition, nous l'avons vu, préalable au désir. Pour Mulvey, donc, seul le travestissement permet à la femme de lier son regard au désir. Encore une fois, il n'y a pas moyen d'expliquer le plaisir de la spectatrice autrement qu'en le conjuguant au masculin, tout comme nous l'avons vu dans les positions théoriques féministes antérieures.

\section{L'écran comme écrin ou l'absence de distance}

La difficulté de conceptualiser la spectatrice, la figure de travesti amenée par Mulvey, a conduit des théoriciennes comme Mary Ann Doane, Tania Modleski, Linda Williams à relancer la question de l'identification de la spectatrice avec l'image des femmes à l'écran en termes d'instabilité, de mobilité, d'ambivalence, de multiplicité. Elles se sont penchées notamment sur des 
films hollywoodiens réalisés dans les années quarante, centrés sur des femmes, s'adressant spécifiquement à un public féminin et mis en marché comme tel. Il s'agissait dès lors de s'interroger sur la nature des plaisirs que les spectatrices prennent dans ce type de cinéma qui prétend justement s'adresser à elles.

Pour Doane, des films comme Caught, Rebecca, Secret Beyond the Door, tous des mélodrames où l'espace domestique est synonyme de menace, de terreur, résistent à une analyse qui se concentre sur la femme comme objet du regard, sur son objectivation comme spectacle selon une structure masculine du regard, bref à une analyse qui met l'accent sur les mécanismes psychiques en relation avec le spectateur masculin (le voyeurisme et le fétichisme) ${ }^{11}$. Mais ces films témoignent d'une grande instabilité dans leur tentative de construire un fantasme féminin et surtout manifestent leur incapacité à soutenir une représentation cohérente de la subjectivité féminine. L'exercice d'un regard actif par l'héroïne est inséparable de sa victimisation, de sa persécution (par le mari, la famille ou l'amant), le désir de regarder se transformant en crainte d'être regardée, en une sorte de "paranoïa narrativisée ", dirait Jacqueline Rose.

Parce que ces films paranoïdes sont dédiés à un public féminin, parce qu'ils prétendent offrir à la spectatrice une identification autre qu'avec le héros masculin actif, ils amènent les femmes à ne pas tomber dans une position de travestie pour ensuite les diriger vers une identification vraiment féminine. Mais comme ces films insistent pour punir les personnages féminins qui manifestent leur désir, ils sont incapables d'offrir aux spectatrices une image "juste" d'elles-même, la première option, l'identification narcissique, devenant aussi difficile. Ce type de woman's film fonctionne donc d'une manière particulièrement complexe rendant impossible une position de lecture confortable pour les femmes, sinon une appréciation favorable de l'image de la femme qu'on lui projette, une image pourtant diminuée.

En s'attardant à des films paranoïdes, Doane en arrive finalement à la même conclusion que Mulvey ou Bellour: le point de vue et le désir féminins sont fortement circonscrits par le regard masculin; le film narratif classique, même centré obsessivement 
sur un personnage féminin, est construit avec un spectateur masculin en tête et le héros agit comme relais dans un processus complexe désigné pour consolider son Moi. Doane s'accorde cependant avec Jacqueline Rose pour dire que ce processus est menacé par la paranoïa latente activée par l'usage du champ I contrechamp. Elle montre d'ailleurs dans son analyse comment cette paranoïa est exacerbée par la tentative des films comme Caught et Rebecca de construire une spectatrice ${ }^{12}$. Finalement, la théoricienne tient à rappeler que le cinéma classique campe le personnage féminin (et implicitement la spectatrice) dans un rôle d'image. La. femme est trop près des apparences; elle n'a d'autre choix que d'accepter la représentation qu'on lui impose d'elle-même ${ }^{13}$. Le spectateur, lui, n'a pas à choisir entre accepter ou rejeter l'image; il peut maintenir une distance. Pour la spectatrice, il y a "surprésence" de l'image; elle est l'image. L'écran devient écrin. La distance nécessaire, condition du voyeurisme dont parle Metz, est absente. Étant donné l'absence de distance, le désir de la spectatrice peut seulement être décrit en termes de narcissisme négatif ${ }^{14}$.

\section{La femme comme image de la perte}

Des théoriciennes comme Tania Modleski ou Linda Williams, qui s'inspirent des travaux de Luce Irigaray, Hélène Cixous et Nancy Chodorow, trouvent carrément que le modèle théorique de Mulvey, par son orientation psychanalytique, est beaucoup trop masculin, et ne fournit qu'une vision en creux de la spectatrice, suggérant des positions identificatoires masochistes ou aliénées. Pour elles, il importe plutôt de spécifier la lecture des spectateurs et des spectatrices d'un film donné et d'attribuer ces différences de lecture en tenant compte des différences dans la construction de l'identité sexuelle.

Ces théoriciennes refusent d'adopter les prémisses freudiennes, modèle où l'homme est posé comme norme, comme point de référence pour définir la femme. On rappelle que les enfants des deux sexes ont la mère comme premier objet d'identification et on met l'emphase sur la relation de la fillette avec sa mère au cours de la phase préœdipienne, comme pilier de son identité, plutôt que la phase œdipienne. La fillette ne brise pas ses liens 
avec sa mère de la même manière que le fait le garçon, ce qui conférerait à l'identité féminine une plus grande fluidité.

Pour Linda Williams, ce modèle d'acquisition de l'identité féminine, soit son potentiel relationnel, contrairement au garçon qui doit se différencier de son objet original d'identification (la mère) afin de prendre son identité masculine, elle l'applique à la lecture que fait la spectatrice d'un mélodrame maternel comme Stella Dallas. Pour elle, le film offre plusieurs points de vue pour l'identification spectatorielle (ceux de Stella, Laurel, Helen, Stephen). Une telle structure narrative s'oppose aux films de "genre" masculin qui sont organisés autour d'un protagoniste masculin contrôlant et dominant le récit. Williams affirme que des genres comme les soap operas, les mélodrames maternels encouragent une identification multiple, plurielle. Alors que l'identification à un seul personnage qui contrôle et domine le récit a pour effet de faire sentir au sujet spectatoriel qu'il a du pouvoir, l'effet d'une identification multiple est de dénuer le spectateur de pouvoir, mais d'augmenter son empathie (faculté de s'identifier à quelqu'un, d'éprouver ce qu'il ressent).

Pour Williams, le mélodrame, en multipliant les points de vue, présente une situation analogue à celle par laquelle la femme construit son identité sous le patriarcat. L'habileté de la femme à entrer en relation, à s'identifier à différentes positions, fait d'elle une lectrice privilégiée du mélodrame. Dans Stella Dallas apparaissent les contradictions entre ce que le patriarcat nous impose (la mère mise "à sa place» et réduite à un rôle de spectatrice) et ce que toute spectatrice empathique ressent (une mère qui perd sa fille et une fille qui perd sa mère).

Pour sa part, Tania Modleski montre que les mélodrames classiques des années trente jusqu'aux années cinquante sont peuplés de personnages mélancoliques, de femmes possédées par un irrésistible désir de retrouver l'objet de leur amour, mais continuellement confrontées à l'impossibilité de réaliser ce désir. Stella Dallas, Lettre d'une inconnue, The Old Maid (Edmund Goulding, 1939) sont des cas exemplaires. Si le mélodrame est associé aux femmes dans la mesure où c'est un genre qui fait de la place aux émotions, si les personnages féminins dans le mélodrame souffrent toujours des affres de l'amour (abandon ou 
perte du mari, de l'amant, des enfants), peut-être que les femmes sont liées à ce genre parce qu'il fournit une porte de sortie (miroir) aux voix féminines refoulées. Peut-être aussi que ces mélodrames ravivent des affects de manque et dévoilent une expérience proprement féminine de la perte. Après tout, ce qu'on retient de ces films, ce sont les moments où les héroïnes renoncent à tout ce qui a eu de l'importance dans leur vie ou encore les événements qui ne se sont jamais produits: le mariage qui n'est jamais survenu, la rencontre ratée, la parole qui n'a pas été dite ${ }^{15}$. Tous ces films engagent des héroïnes sur une voie mélancolique (angoisse de séparation, agir dépressif, perte d'estime de soi et appauvrissement du Moi), visent à restaurer l'objet perdu, mais ne réussissent pas à combler le vide, à éluder le manque. Ils ne réussissent qu'à l'activer. Bref, ils engagent une posture mélancolique, en favorisant des affects d'absence, de perte et d'abandon.

Cette trajectoire qui pose des différences de lecture selon le sexe en tenant compte de la façon qu'ont les hommes et les femmes de construire leur identité sexuelle sous le patriarcat n'est pas au-dessus de tout soupçon. Pour Diane Waldman, par exemple, même dans le cas où l'on trouve différentes lectures, doit-on l'attribuer seulement à la différence sexuelle? Que faiton des différences de classe, de race, d'orientation sexuelle, des spécificités culturelles et historiques? Ces questions prendront d'ailleurs le haut du pavé des études féministes du cinéma au milieu des années quatre-vingt avec l'arrivée en force des cultural studies. Non seulement elles compliquent la question d'identification au cinéma, mais elles conduisent presque inévitablement à une ambivalence des positions identificatoires du sujet spectatoriel, poussant «[...] jusqu’à leur dernier retranchement l'identification des différences" (Bellemare, 1996, p. 53) ${ }^{16}$.

Par ailleurs, si pour plusieurs théoriciennes féministes, le cinéma classique exige de la spectatrice qu'elle se convertisse à ce qu'il représente, voire qu'elle aime l'image qu'on projette d'elle-même, une image pourtant diminuée, Kaja Silverman ouvre une brèche dans les théories du genre en affirmant qu'il ne faut pas trop prendre au pied de la lettre (et de l'image) le signifié fictionnel du 
corpus filmique classique: son spectacle du manque féminin ad nauseam est un leurre, en cache un autre plus fondamental ${ }^{17}$.

\section{Un manque qui en cache un autre}

Labsence que le cinéma situe sur le corps féminin a son origine ailleurs dans les opérations du signifiant (cinématographique). L'équation liant la femme et le manque serait donc une construction secondaire, une construction couvrant un sacrifice antérieur, soit la castration symbolique ou la perte de l'objet, castration d'ailleurs à laquelle tous les sujets culturels sont soumis, puisqu'elle coïncide avec la séparation d'avec le monde des objets et avec l'entrée dans le langage. Comme le précise Silverman, s'il y a une crise qui entoure la découverte que la construction filmique est organisée autour de l'absence, c'est parce que le sujet regardant est aussi organisé autour de la même absence. L'histoire du sujet (il ou elle) se déploie à travers une série de divisions et de pertes, la première de ces divisions survenant au stade du miroir, au moment où l'enfant se perçoit pour la première fois. L'enfant aperçoit alors les objets par excellence qui lui appartiennent: sa mère, mais aussi sa propre image. Il se voit comme un autre à côté d'un autre. L'un lui garantit que l'autre, c'est bien lui. Donc, la subjectivité de l'enfant dépend de cette reconnaissance de la distance qui le sépare de l'autre. De même, l'entrée dans le langage se fait au moment où l'objet est irrémédiablement perdu. Le langage prend la place du réel pour le sujet. Donc, le sujet masculin, comme le sujet féminin, est déjà structuré par une absence avant le moment même où s'inscrit la différence anatomique.

Autrement dit, souligne Silverman, le cinéma ravive chez le spectateur le désir primordial de l'objet perdu - mais en vient toujours à le décevoir - et peut réactiver à tout moment le traumatisme de la perte, de la castration symbolique. Ce traumatisme, précisent pour leur part Oudart et Dayan, survient à chaque fois que le spectateur reconnait son impuissance " discursive ", comprend qu'il ou elle est seulement autorisé à voir ce qui survient dans l'axe du regard de l'autre qui est absent. À ce titre, le succès du cinéma classique peut être mesuré à sa capacité à construire des substituts adéquats, à compenser le réel absent, 
à renforcer l'illusion chez le spectateur qu'il est à la fois le centre, la source et le sujet des émotions que lui procure le film.

Mais attention! rappelle Silverman. Cette perte qui structure le cinéma et affecte son spectateur, cette crise qui survient à chaque fois qu'on lui rappelle sa subordination visuelle et auditive (à cet Autre transcendantal) est recouverte par une autre crise, le drame diégétique de la castration (celle décrite justement par Mulvey) avec la représentation de l'homme "phallique» et de la femme " mutilée ". Autrement dit, pour dissimuler cette perte fondamentale de l'objet, le cinéma classique met en place la différence sexuelle comme moyen de défense, moyen qui ne sert en fait qu'à protéger le spectateur masculin.

Silverman conclut que ce manque associé au sujet féminin doit être lu comme un symptôme de la condition masculine. Plus, le cinéma dominant initie et orchestre le transfert des pertes qui affectent le sujet masculin vers la femme par une série d'activités défensives dont le désaveu, le fétichisme, le voyeurisme, le sadisme et la projection. Il projette le manque masculin dans des personnages féminins sous l'apparence d'une déficience anatomique et d'une incapacité discursive. Cette narrativisation à répétition de la castration entraine l'exclusion de la femme du pouvoir symbolique, exclusion qui se traduit par une relation passive du personnage féminin au régime scopique et auditif.

Le recours à la technique du champ / contrechamp, par exemple, aligne le corps féminin sur le regard masculin. Ces deux plans font en sorte que le spectateur, via les personnages du film, retrouve sa puissance visuelle et auditive. Autrement dit, la subordination du spectateur à un "Autre transcendantal" est couverte par l'inscription dans la diégèse d'un personnage (masculin en l'occurrence) d'où les images et les sons semblent défiler, un personnage avec une vision, une écoute et un discours qui font autorité, et avec lequel le spectateur s'identifie. Ce qu'il faut retenir ici, c'est jusqu’à quel point la représentation compensatoire est codée comme masculine dans le cinéma narratif classique, jusqu’à quel point le dispositif cinématographique emprunte une forme paternelle, jusqu'à quel point la subordination visuelle et narrative de la femme est le pivot qui permet au dispositif cinématographique de fonctionner. 
Bref, le cinéma classique organise le flot des images et des sons pour protéger le sujet masculin de cette rencontre déplaisante avec soi: sa propre castration. Le corps féminin comme la voix féminine fonctionnent comme un fétiche dans la mesure où ils recouvrent la face indicible de la subjectivité masculine; les femmes au cinéma sont contraintes d'exhiber le manque et protègent ainsi le sujet masculin en absorbant ses pertes aussi bien que celles qui structurent la subjectivité féminine. Cela se traduit par la représentation d'un sujet féminin incapable de voir, d'entendre ou de parler de manière autoritaire. Même que le regard féminin manifeste si peu de résistance au regard masculin qu'il semble une sorte d'extension de celui-ci (son regard est central dans la mesure où il doit s'aligner avec son vis-à-vis masculin). Pire, la voix féminine révèle une formidable propension à se dénigrer. Pas de la place à une voix féminine désincarnée dans le corpus classique ${ }^{18}$ ! La voix off, au même titre que le regard, constitue un mécanisme par lequel le cinéma classique parvient à donner aux personnages masculins l'autorité énonciative. Dépouillé de son enveloppe corporelle, dissocié du visible, le sujet masculin peut parler à partir d'une position d'autorité, s'enligner du côté du symbolique, bref passer du côté du signifrant. Autant le sujet féminin dans le cinéma classique est défini par sa visibilité, sa corporalité, sa spécularité, sa présence, autant le sujet masculin est dissocié du visible et associé à l'autorité énonciative, à la connaissance, au pouvoir, à la Loi.

\section{Place à la voix comme créatrice de distance}

Face à un tel régime scopique où l'écran se fait écrin pour la femme et où son corps est sous étroite surveillance, il n'est pas surprenant que bon nombre de réalisatrices aient tablé sur une voix féminine désincarnée, délestée de son enveloppe corporelle, et opté pour la dissonance et la fragmentation. Qu'on pense aux films de Marguerite Duras, Chantal Akerman ou Lizzie Borden. Ces réalisatrices ont fracturé la diégèse, multiplié les voix féminines de façon telle qu'il est impossible de leur donner un ancrage corporel. Elles ont réussi à créer ce que le cinéma classique ne peut pas faire pour la spectatrice: le maintien d'une distance d'avec l'image, une condition non seulement préalable à la 
subjectivité et au langage mais aussi au désir. India Song de Duras (1975), par exemple, à travers le déploiement de plusieurs voix off et de leur relation désirante à une image féminine inaccessible - une femme qui, le film le dit, est déjà morte - met en scène une représentation qui table sur le manque, la perte et la distance. Le film déploie un fantasme de perte et de distance, un fantasme de désir, un désir qui ne peut être satisfait. Il laisse ouverte la question du désir et d'une position féminine en relation avec ce désir.

Puisons un autre exemple cette fois dans le corpus filmique d'Akerman: Jeanne Dielman (1975). Ce film mise sur l'absence de raccords et la présence de longs plans-séquences, bref sur une sorte d'épure où les gestes répétitifs du personnage féminin prennent tout l'espace de la vision. Le sujet de la réception n'est pas inclus dans ce film à travers le regard des personnages; le film offre à la spectatrice la possibilité de se distancier par rapport à l'image du corps féminin représenté, un corps qui n'est pas montré comme ce que le personnage féminin ou la spectatrice devrait devenir. Garder ses distances, maintenir un écart entre l'image et soi, n'est-ce pas répondre à une des conditions préalables au plaisir spectatoriel stipulé par Metz?

Dernier exemple avant de conclure: Born in Flames de Lizzie Borden (1983). Comme le précise Teresa de Lauretis, ce qui frappe dans ce film souvent cité par les théoriciennes féministes, c'est le fait qu'il s'adresse au sujet spectatoriel comme femme, pose la question des différences entre femmes, multiplie les points de vue, bref construit le sujet féminin dans son hétérogénéité et sa multiplicité plutôt que d'en brosser une image positive ou négative"?

En somme, les films de Duras, d'Akerman ou de Borden engagent une transformation de la vision, en inventant des formes et procès de représentation d'un sujet social: les femmes. Maintenir une distance avec l'image pour la spectatrice, déployer des stratégies d'adresse et surtout dépouiller la voix féminine du corps ${ }^{20}$ seraient donc aux yeux de plusieurs théoriciennes féministes modifier de fond en comble le régime scopique à la base de la représentation de la femme dans le cinéma classique, puisque c'est précisément comme corps, comme pré- 
sence que la femme est construite. N'est-ce pas le vœu que formulait Mulvey à la fin de son essai choc en 1975?

Depuis une vingtaine d'années, les gender studies s'attardent au dispositif cinématographique, font ressortir tout un paradigme de négativités autour de l'image de la femme au cinéma et de l'identité de la spectatrice. Après avoir défini la spectatrice par la négative ou comme absence, on a remis en question l'homogénéité du spectateur, le modèle un peu trop masculin de Laura Mulvey qui fournit une vision en creux de la spectatrice. Si on a fait une place à la spectatrice, cette dernière est souvent condamnée à une posture insoutenable, prise au milieu d'images qui reflètent son exclusion, sa fragmentation. Plus souvent qu'autrement, la spectatrice ne peut maintenir de distance d'avec l'image qu'on projette d'elle-même, une image pourtant diminuée. Plus rarement, elle y trouve un terrain de reconnaissance, de plaisir ou de désir. Avec le temps, les études féministes en cinéma ont compliqué l'analyse de la spectatrice en ajoutant les différences de classe, de race, de culture à la différence sexuelle pour rendre compte de la complexité du processus d'identification au cinéma. Par ailleurs, les écrits de Silverman sur la subjectivité masculine ouvrent une brèche sur les théories du genre en montrant comment les femmes dans le cinéma classique sont contraintes d'exhiber le manque afin d'absorber les pertes qui affectent le sujet masculin. Il ne s'agit plus de faire du cinéma classique un mauvais objet, comme plusieurs théoriciennes ont eu tendance à le faire, mais de le voir comme un dispositif qui se nourrit de l'absence, de la perte et qui réécrit, ici, ces négativités sur le corps féminin. Et cette absence ne pourra jamais être abolie, au contraire. Par ailleurs, face à un régime scopique où le corps féminin est pris dans un étau, sous étroite surveillance, des films comme ceux d'Akerman ou de Duras réussissent à créer ce que le cinéma classique ne peut pas faire pour la spectatrice: le maintien d'une distance d'avec l'image, une mise en scène qui table sur l'absence du corps féminin comme point d'ancrage de l'identification, et la construction d'un désir féminin. 


\section{NOTES}

1 Phase au cours de laquelle l'enfant découvre sa propre image et l'image de l'autre, et constitue imaginairement son unité corporelle. La position du spectateur ressemble à celle de l'enfant au miroir telle que décrite par Lacan. Le dispositif cinématographique reproduit partiellement les conditions qui ont présidé, dans la petite enfance, à la constitution imaginaire du Moi lors de la phase du miroir. Cela dit, rappelle justement Mary Ann Doane, il y a tout de même une différence entre la phase du miroir et la situation du sujet spectatoriel. Contrairement au miroir, l'écran renvoie tout sauf l'image du propre corps du spectateur...

2 Expression tirée de sa thèse de doctorat, La Mélancolie et le banal. Essai sur le cinéma québécois (Paris: Université Paris III-Sorbonne Nouvelle, 1992).

3 Les films de Fritz Lang (Secret Beyond the Door, 1948), Howard Hawks (Gentlemen Prefer Blondes, 1953), Josef von Sternberg (Morocco, 1930), Michael Curtiz (Mildred Pierce, 1945), Alfred Hitchcock (Rebecca, 1940; Les Oiseaux, 1963), King Vidor (Stella Dallas, 1937; Duel in the Sun, 1946), Charles Vidor (Gilda, 1947), Raoul Walsh (Band of Angels, 1957; The King and Four Queens, 1956), Max Ophüls (Caught, 1940; Letter from an Unknown Woman, 1948; Madame de, 1953), Stanley Kramer (Not as a Stranger, 1955) sont là pour rappeler le sort réservé au "spectre féminin ".

4 Pour Cook et Johnston, Band of Angels (L'esclave libre, 1957) reconduit de façon exemplaire l'opposition masculin/non-masculin dans le cinéma classique. Le film raconte l'histoire d'une fille de planteur du Kentucky qui découvre à la mort de son père ruiné que sa mère était une esclave noire. Seule, elle se retrouve au marché des esclaves et est acheté par un riche gentilhomme (Clark Gable). L'histoire se déroule sur fond de guerre civile américaine. La première partie du film illustre une tranche de vie de l'héroïne, son changement de statut (le passage de lady à esclave). Au milieu du film apparaît le héros qui l'achète au marché des esclaves. À partir de ce moment, le personnage féminin devient celui par qui l'homme peut interagir avec les autres, donner sens à son passé, acquérir une connaissance de soi, devenir sujet du désir. Chez Raoul Walsh donc, la femme occupe une place centrale dans la mesure où cette place est assignée dans la logique du désir masculin. Non seulement elle est un objet d'échange - "[...] a token exchange in this patriarchal order" - , mais sa place est définie " $[\ldots]$ as the locus of "lack", an empry space which must be filled in the working through of man's desire to find his own place in society" (Pam Cook, "Approaching the Work of Dorothy Arzner ", dans Claire Johnston (direction), The Works of Dorothy Arzner: Towards a Feminist Criticism. Londres: British Film Institute, 1975, p. 47).

5 Dans les films d'Arzner, il y a une volonté de transgression de la part des femmes, d'aller au-delà de la place ou du rôle qui leur est assigné dans la société patriarcale. Elles élaborent leur identité par désir et par transgression, recherchent une existence indépendante en dehors du discours masculin. Même si en bout de ligne le personnage féminin n'a pas réussi à imposer son désir, son discours donne cohérence au texte même du film et par là lézarde l'idéologie sexiste, patriarcale.

6 Bellour analyse un segment de 81 plans: Melanie Daniels se rend en bateau à Bodega Bay pour offri: à Cathy, petite sour de Mitch Brenner, un avocat qu'elle connaît à peine, un couple d'«inséparables», puis revient au quai du village. Sur le chemin du retour, elle se fait attaquer par une mouette. Bellour montre comment le segment progresse via l'orchestration de trois systèmes binaires: voyant/vu (point de vue), proche/lointain (cadrage), mouvement/non-mouvement. Il analyse en détail le jeu des regards entre l'homme et la femme et les mécanismes d'identification. Un regard de Melanie est le moteur de ce segment. Le désir dans son regard est nécessaire à la fiction, mais seulement parce qu'il sera finalement assujetti au regard masculin.

Le spectacle du "manque féminin" au cinéma: un leurre qui en cache un autre 
7 Voir Janet Bergstrom, "Enunciation and Sexual Difference", Camera Obscura 3-4 (1979), p. 33-69 et "Alternation, Segmentation, Hypnosis: Interview with Raymond Bellour ", Camera Obscura 3-4 (1979), p. 71-103.

8 Le dispositif cinématographique fait en sorte que le spectateur a la sensation que c'est lui le "grand imagier". Dans le cinéma narratif classique, le travail minuticux et invisible de l'énonciation entretient l'impression chez le spectateur qu'il entre de luimême dans le récit et renforce l'illusion qu'il est à la fois le centre, la source et le sujet des émotions que lui procure le film - comme dans l'expérience onirique. Plus, le plaisir voyeuriste au cinéma s'est manifesté historiquement dans l'image de la femme. Le dispositif cinématographique s'avère donc un site institutionnel où l'appropriation masculine de la conduite scopique a imposé une position et une fonction à la femme: elle est une image et un objet absent.

9 Voir à ce sujet l'article de Jackie Stacey, "Desperately Seeking Difference", Screen, vol. 28, n' 1 (1987), p. 50. Dans cet article, Stacey s'attarde à deux films qui mettent en scène un sujet féminin désirant au cœur du récit, pour analyser les phénomènes de désir et d'identification entre femmes lorsque celles-ci figurent à la fois comme sujet et objet de désir. Les deux films retenus sont: All About Eve de Joseph Mankiewicz (1950), un récit hollywoodien classique, et Desperately Seeking Suzan de Susan Seidelman (1984), une production américaine indépendante. Stacey se demande ce qui se passe lorsque c'est une femme au lieu d'un homme qui prend plaisir à regarder une autre femme et lorsque c'est une femme qui fait avancer le récit. Elle fait ressortir les plaisirs qui s'offrent à la spectatrice et qui ne sauraient être réduits à une simple identification au personnage féminin, puisque ces films insistent également sur les différences entre femmes.

10 Le film raconte l'histoire d'une jeune Métis, Pearl, aimée par deux frères, Lewt et Jesse. Ceux-ci représentent les deux aspects de son désir, personnifiant son drame intérieur. D'un côté, on trouve Jesse, celui qui témoigne de son amour pour la culture, l'éducation, l'argent et qui est destiné a être gouverneur de l'État, bref celui qui représente le "bon chemin" pour Pearl, la conduisant sur la voie de la sexualité passive et lui apprenant à être une lady. De l'autre, on trouve Lewr, celui qui aime les chevaux, les fusils, le hors-la-loi par excellence, bref celui qui représente le "mauvais chemin" pour l'héroïne, celui de la passion, une passion sexuelle non basée sur la maturité, mais sur la régression. Avec Lewt, Pearl peut être un "garçon manqué " (elle peut galoper, nager, tirer du fusil). Mais en dernier ressort, il n'y a pas de place pour Pearl dans l'univers de Lewt, un univers machiste, pas plus quil n'y en aura pour elle dans celui de Jesse. Le film consiste en une série d' "oscillations" entre deux voies tout aussi désespérées pour l'héroïne.

11 Pour Doane, les théories du cinéma se sont peu préoccupées de construire une spectatrice. Le spectateur anticipé et positionné par le texte, défini par les mécanismes psychiques de voyeurisme, de fétichisme et d'identification primaire, est inévitablement masculin. Ainsi, les films hollywoodiens sont vus comme des "structures compensatoires conçues pour défendre la psyché masculine contre la menace que représente l'image de la fermme". Bien que ces analyses filmiques aient permis de comprendre le fonctionnement du cinéma narratif classique, elles ne font que répéter cette tendance historique de la psychanalyse à se pencher sur la psyché masculine aux dépens de celui de la femme. Dans un contexte théorique où le cinéma est vu comme un appel au voyeurisme et au fétichisme, est-il possible de construire une spectatrice? Cela se pose d'autant plus quand il s'agit de considérer les films hollywoodiens des années quarante qui prétendent s'adresser aux femmes. Voir Mary Ann Doane, "The "Woman's Film": Possession and Address", dans Mary Ann Doane et al. (direction), Re-Vision. Frederick: University of Publications of America (1984), p. 67-82 et "Caught and Rebecca: The Inscription of Femininity as Absence", dans Constance 
12 Dans Rebecca, signale Doane, on trouve une scène cruciale où le désir d'être regardé du personnage féminin est transformé en crainte d'être regardé. Il s'agit de la séquence de projection du film amateur réalisé par Maxim, le mari de l'héroïne. Cette mise en abyme (le film dans le film) met en évidence l'incapacité du film de soutenir la représentation d'un sujet féminin désirant et récupère l'image du désir féminin présent tout au long du film en ramenant une image de la femme plus conforme à ce qui nous est habituellement montré (l'image du couple standard). Attardons-nous un instant à cette séquence qui rend la position d'une spectatrice inconfortable, voire impossible.

La séquence s'ouvre sur l'héroïne (jouée par l'actrice Joan Fontaine; on ne connaîtra jamais le nom de son personnage) pénétrant dans le bureau de son mari, Maxim, dans l'espoir de lui plaire. Elle est habillée en robe de soirée, exactement comme dans le magazine de mode qu'elle feuilletait deux plans auparavant. Comme spectateur, nous avions eu droit tout juste avant à la séquence du film amateur à deux plans fixes, montrant des illustrations de la revue. Le film élide le plan qui aurait identifié le personnage féminin à une spectatrice, la transformant immédiatement en une image - celle du magazine - une image visant à séduire son mari. Lhéroïne donc "entre au cinéma" dans l'espoir de devenir un spectacle pour son "homme", mais elle est vite reléguée à la position de spectatrice aliénée, spectatrice d'images que son mari préfere retenir d'elle, celles prises lors du voyage de noces.

Dans cette séquence toujours, Maxim contrôle la projection et l'éclairage. Le film amateur montre des moments forts de leur voyage de noces filmés par Maxim. L'image de Rebecca dans ce film amateur constitue un déni de l'image qu'elle s'est construite pour plaire à son mari. Le film projeté est interrompu à deux reprises, déplaçant l'investissement spectatoriel de l'écran à la femme. La première interruption est causée par un bri de pellicule qui cö̈ncide avec une remarque de Rebecca : "J'espère que notre lune de miel durera toujours. "Pendant que Maxim tente de fixer la pellicule, un serviteur entre et signale qu'il a découvert les morceaux d'un bibelot (un "Cupidon!") dans un tiroir, bibelot qu’avait cassé et caché Rebecca pour éviter les remontrances. Cette pause forcée dévoile l'incapacité de l'héroïne d'assumer sa position de maîtresse de Manderley, bref de remplacer Rebecca, le personnage fantomatique qui hante le récit et subjugue Fontaine. La projection du film reprend, mais sera interrompue à nouveaı quand l'héroïne suggère que son mari doit l'avoir mariée pour éviter les commérages. Maxim marche alors abruptement vers elle, bloquant l'image projetée avec son corps et "castrant" son regard. Se substituant à l'écran, il lance un regard agressif en direction de la caméra, retournant le regard de Fontaine contre ellemême. La terreur que suscite cette violente réorganisation cinématographique des regards est évidente dans les yeux du personnage féminin, la seule partie du visage éclairée partiellement par la lumière de l'écran, contrastant avec les images claires, cohérentes, homogènes du film-maison qui dévoilent la stabilité et l'homogénéité du couple. Linterruption du film conduit à la libération de tendances agressives, le désir d'être regardé du personnage féminin se transformant en crainte d'être regardé.

13 Dans Caught et Rebecca, il est significatif que les deux héroïnes ne peuvent se distancer des images de femmes qu'elles trouvent dans des revues de mode (d'un côté, une femme en manteau de vison; de l'autre, une femme en robe de satin et avec collier de perles). Pour ces deux femmes, posséder l'image (la photographie dans le magazine de mode), c'est devenir cette image.

14 Pour Doane, cette opposition proximité/distance est plus cruciale que l'opposition passivité/activité amenée par Mulvey dans la problématique de la différence sexuelle au cinéma. Aussi suggère-t-elle dans des articles ultérieurs de concevoir 
théoriquement la position de la spectatrice en termes de mascarade, une façon de s'approprier cette nécessaire distance entre elle-même et l'image (essentielle dans la constitution du Sujet en psychanalyse comme dans l'opération sémiotique). Comme le précise Doane, porter un masque, c'est créer un vide, une distance entre soi et sa propre image, et lire différemment la féminité. Voir Mary Ann Doane, "Film and the Mas-

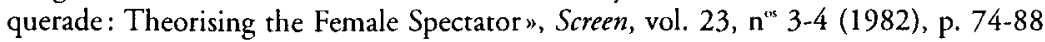
et "Masquerade Reconsidered: Further Thoughts on the Female Spectator", Discourse, vol. 11, n" 1 (1988-1989), p. 42-54.

15 Par exemple, dans Lettre d'une inconnue, Lisa tait son amour pour Stefan et cache l'existence de son fils toute sa vie durant. De même, Stella Dallas, dans le film éponyme, reste anonyme derrière une grille de fer observant le mariage de sa fille à travers la fenêtre. Son amour démesuré pour sa fille doit être brimé.

16 Je ne pourrai malheureusement pas aborder dans le cadre de cet arricle cetre trajectoire qu'ont prise les études féministes au milieu des années quatre-vingt, faute d'espace et compte tenu de mon orientation de départ: soit l'étude d'une série de textes féministes fondateurs en cinéma, qui se sont arrimés à la psychanalyse et qui ont participé à une meilleure connaissance du signifiant cinématographique, du travail spécifique du cinéma classique et des différentes strates de négativités autour du personnage féminin. Pour en savoir davantage sur l'orientation qu'ont prise les études féministes du spectatorat, voir notamment l'article de Teresa de Lauretis, "Aesthetic and Feminist Theory: Rethingking Women's Cinema ", New German Critique, n" 34 (1985), p. 154-175, de même que le numéro spécial de Camera Obscura intitulé "The Spectatrix ", n" 20-21 (1989).

17 Je reprendrai dans les pages suivantes les principaux arguments développés par Kaja Silverman dans The Acoustic Mirror: The Female Voice in Psychoanalysis and Cinema (Bloomington : Indiana University Press, 1988).

18 Le cinéma classique cheville la voix féminine au corps. Il est rare qu'on présente une voix de femme séparée du corps qui la supporte. Quand c'est le cas, elle a le même statut que la voix off du personnage masculin dans le film noir : "It is autobiographical, evoking in a reminiscent fashion a fiction whithin which the speaker figures centrally as a bodily presence" (Kaja Silverman, The Acoustic Mirror: The Female Voice in Psychoanaiysis and Cinema. Bloomington: Indiana University Press, 1988, p. 165). Le personnage féminin est confiné dans le champ de vision et d'audition de l'homme. Permettre à un personnage féminin d'être vu sans être entendu serait activer les codes herméneutique et culturel qui définissent la femme comme une énigme, inaccessible à une interprétation masculine. De même, permettre à un personnage féminin d'être entendu sans être vu semblerait aussi périlleux, puisque cela la mettrait hors d'atteinte du regard masculin et la libérerait des obligations que fait valoir le regard masculin.

19 Pour plus de détails, voir l'article de Teresa de Lauretis, "Aesthetic and Feminist Theory: Rethingking Women's Cinema ", New German Critique, n" 34 (1985), p. 154-175.

20 Cette position va à l'encontre des travaux psychanalytiques de Julia Kristeva ou Luce Irigaray qui situent la féminité dans le royaume d'une authentique proximité et d'une irrésistible présence à soi. Qu'on pense notamment à la position de Luce Irigaray sur une parole et une écriture féminine chevillées au corps féminin ("embodied speech or writing"). Irigaray croit possible d'élaborer un nouveau langage pour les femmes capable de coexister avec leur corps aussi étroitement que les "deux lèvres de la vulve", bref un modèle langagier analogue au modèle corporel, avec les mêmes caractéristiques, à savoir la proximité, la contiguiité, la simultanéité. Ce modèle va tout à fait l'encontre du modèle linguistique proposé par Lacan, qui table sur la perte du corps, du référent comme prix à payer pour accéder au signifiant. Pour Silverman, 
ce paradigme qui tente de dénier la relation fondamentalement arbitraire du langage au référent est impossible à soutenir. Cela ne veut pas dire que le projet féministe ne doive pas tenter de "réécrire le corps". Au contraire! Cela demeure un point crucial. Cependant, l'enjeu devrait être la transformation des conditions discursives à travers lesquelles les femmes vivent leur corporalité.

\section{OUVRAGES CITÉS}

Bellemare, Denis. "Narcissisme et corps spectatoriel ». Cinémas, vol. 7, n" 1-2 (1996), p. 37-54.

Bellemare, Denis. La Mélancolie et le banal. Essai sur le cinéma québécois (thèse de nouveau doctorat). Paris: Université Paris III-Sorbonne Nouvelle, 1992.

Byars, Jackie. "Gazes / Voices / Power: Expanding Psychoanalysis for Film and Television Theory", dans E. Deidre Pribram (direction), Female Spectators. Looking at Film and Television. Londres: Verso (1988), p. 110-131.

Cook, Pam. "Approaching the Work of Dorothy Arzner", dans Claire Johnston (direction), The Works of Dorothy Arzner: Towards a Feminist Criticism. Londres: British Film Institute (1975), p. 9-18.

Cook, Pam et Johnston, Claire. "The Place of the Woman in the Cinema of Raoul Walsh", dans Phil Hardy (direction), Raoul Walsh. Colchester: Vineyard Press (1974), p. 93-110.

Johnston, Claire. "Women's Cinema as Counter-Cinema », dans Patricia Erens (direction), Sexual Strategem: New York: Horizon Press (1979), p. 133-143.

Johnston, Claire. "Dorothy Arzner: Critical Strategies ", dans Claire Johnston (direction), The Works of Dorothy Arzner: Towards a Feminist Criticism. Londres: British Film Institute (1975), p. 36-45.

Metz, Christian. Le Signifiant imaginaire. Psychanalyse et cinema. Paris: Christian Bourgois, 1993.

Mulvey, Laura. "Visual Pleasure and Narrative Cinema". Screen, vol. 16, n" 3 (1975) p. 6-18.

Rose, Jacqueline. "Paranoia and the Film System", dans Constance Penley (direction), Feminism and Film Theory. New York: Routledge (1988), p. 41-58.

Rose, Jacqueline. "The Cinematic Apparatus: Problems in Current Theory", Sexuality in the Field of Vision. Londres: Verso (1986), p. 199-214.

Silverman, Kaja. The Acoustic Mirror: The Female Voice in Psychoanalysis and Cinema. Bloomington : Indiana University Press, 1988. 